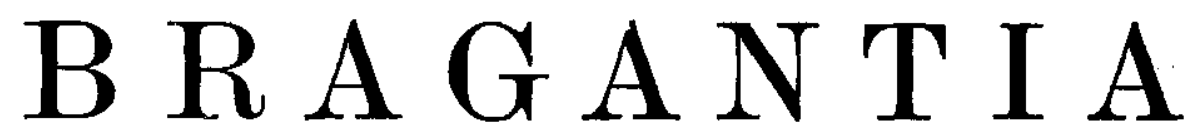

Revista Cientifica do Instituto Agronômico do Estado de São Paulo

Vol. 33

Campinas, maio de 1974

N. ${ }^{\circ} 5$

\title{
COMPORTAMENTO DE CULTIVARES DE TRIGO EM CONDIÇÕES DE SEQUEIRO NO ESTADO DE SÃO PAULO $\left({ }^{1,2}\right)$
}

Carlos Eduardo de Oliveira Camargo $\left(^{3}\right)$, Seção de Arroz e Cereais de Inverno, Mílton Alcovér, Estaçâo Experimental de Capão Bonito, Instituto Agronômico, e Eduardo Issa ('), Seção de Doenças das Plantas Alimenticias Básicas e Olericolas, Instituto Biológico

\section{SINOPSE}

Em ensaios de competição regional de cultivares de trigo em condição de sequeiro, efetuados no ano de 1971, no Estado de São Paulo, destacaram-se os seguintes, na ordem decrescente de produtividade: S-12, IRN-526-63, Pitic-62, $\mathrm{BH}-1146$, IAC-5 e IAC-7. As diferenças de produçōes desses cultivares não foram significativas (Tukey a $5 \%$ ). O cultivar IRN-526-63, de origem mexicana, apresentou também resistência à ferrugem do colmo e ausência de acamamento, aliados a boa adaptação às diferentes regiōes triticolas paulistas em condição de sequeiro.

\section{1 - INTRODUÇÃO}

O estudo de cultivares de trigo para o Estado de São Paulo vem sendo realizado há vários anos na Estação Experimental de Capão Bonito, Instituto Agronômico, onde foram selecionados numerosos cultivares adaptados às nossas condições, entre os quais IAC-5, que já se encontra, com sucesso, em cultivo comercial.

Por outro lado ,a Seção de Doenças das Plantas Alimentícias Básicas e Olerícolas, Instituto Biológico $(\mathbf{3}, \mathbf{4})$, vem realizando, anualmente, o Ensaio Internacional de Ferrugem de Trigos de Primavera, cujo objetivo é a obtenção de material de alta qualidade agronômica

(1) Recebido para publicacão em 4 de fevereiro de 1974.

(2) Os autores agradecem aos Engenheiros Agrónomos Armando Pettinelli, Ary de Arruda Veiga, Derly Machado de Souza, Joăo Aloisi Sobrinho e Sebastiāo Alves a colaboraçāo na realizaçăo deste trabalho.

(a) Com botsa de suplementaçăo do CNPq. 
resistente às principais doenças do trigo, como a ferrugem do colmo (Puccinia graminis tritici) e a ferrugem da folha (Puccinia recondita).

Camargo $(1,2)$, em 1969, tendo plantado nove experimentos com 36 cultivares, em diferentes regiōes do Estado de São Paulo, verificou que o IRN-526-63 foi superior aos demais cultivares estudados quanto à produtividade e resistência às doenças, principalmente à ferrugem do colmo.

Outros oito experimentos do mesmo autor (2) mostraram que os cultivares S-12, IRN-526-63, IAC-5, IAS-51 (Albatroz) foram superiores, quanto à produção, em relação ao cultivar $\mathrm{BH}-1146$, que está sendo cultivado há vários anos em caráter comercial no Estado de São Paulo.

No presente trabalho procurou-se estudar com mais detalhes o comportamento agronômico de nove cultivares de trigo, avaliando-se dados de produção, ciclo, altura, resistência à ferrugem do colmo, ferrugem da folha e Helminthosporium sp., acamamento, comprimento da espiga, número de espiguetas por espiga, número de grãos por espiga, número de grãos por espigueta e peso de 1000 grãos.

\section{2 - MATERIAIS E MÉTODOS}

A relação e a origem dos nove cultivares estudados encontram-se a seguir:

BH-1146 - Selecionado no Instituto Agronômico de Minas Gerais, Belo Horizonte, e proveniente do cruzamento Ponta Grossa I x Fronteira, híbrido esse que foi cruzado com o cultivar Mentana. O cultivar BH-1146 foi utilizado como controle, por ser o mais cultivado comercialmente na ocasião, no Estado de São Paulo.

IAC-5 ou Maringá - Oriundo da progênie 16494, proveniente de seleçāo do hibrido entre a progênie 7124 e o cultivar Polissu $\left(\mathrm{PG}_{1}\right)$, em 1956, na Estação Experimental de Capão Bonito. A 7124 resultou de seleção do hibrido entre os cultivares Frontana e Kenya, em 1951.

S-12 - Introduzido pelo Instituto Biológico, oriundo da Secretaria da Agricultura do Estado do Rio Grande do Sul, criado na Estação Experimental de Júlio de Castilhos e obtido por seleção do cruzamento do híbrido entre os cultivares Veranópolis e Mayo 54 e o Veranópolis.

S-33 - Originoul-se da progênie 16429, que foi criada na Estaçăo Experimental de Capão Bonito, a partir de seleção, realizada em 1956, do H-416, 
híbrido derivado do cruzamento entre a progênie 7124 e o cultivar Ponta Grossa I. A progénie 7124 , por sua vez, é uma seleção do híbrido entre o cultivar Frontana e um sem nome, procedente de São Paulo.

IAS-51 ou Albatroz - Introduzido pelo Instituto Agronômico sob o número I-29815, no ano de 1964, do Instituto de Pesquisas e Experimentação Agropecuária do Sul, Pelotas, Rio Grande do Sul.

IRN-526-63 e Pitic-62 - Selecionados no Centro Internacional de Melhoramento de Milho e Trigo (CIMMYT), no México, e introduzidos através do Ensaio Internacional de Ferrugem de Trigo de Primavera, sob os números 526 e 464, respectivamente, no ano de 1973, pela Seção de Doenças das Plantas Alimenticias Básicas e Olerícolas, Instituto Biológico (figura 1).

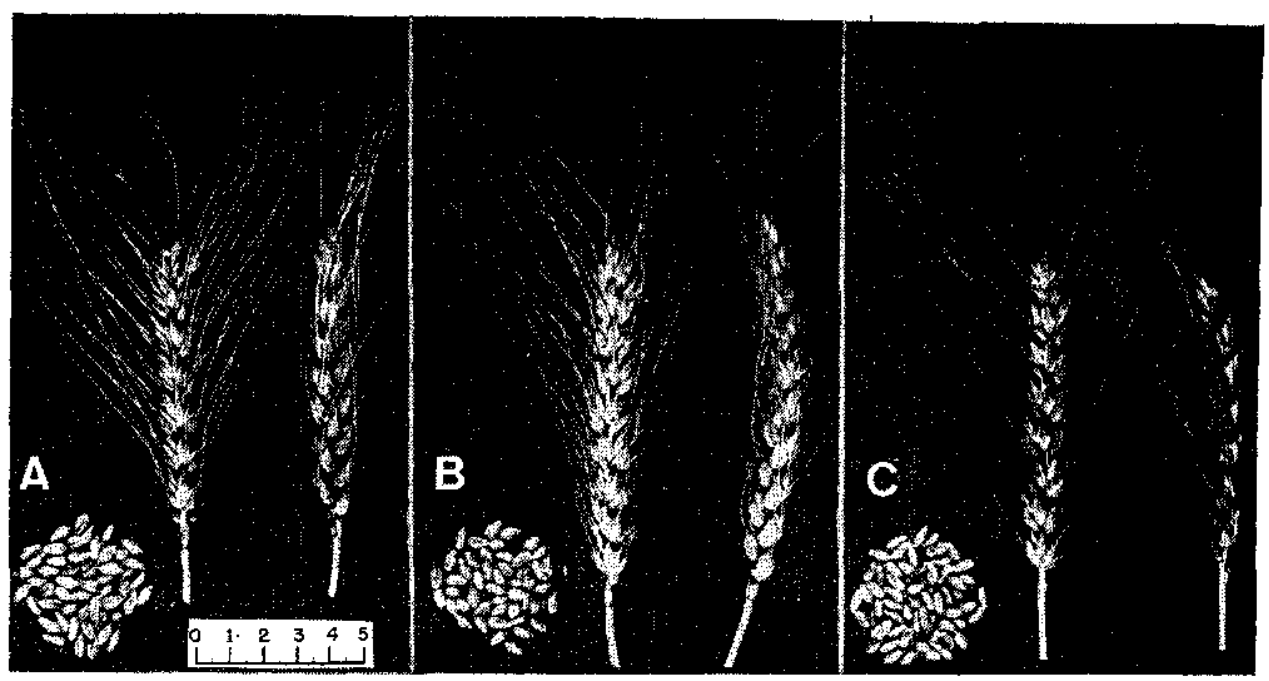

Figura 1. - Espigas e grãos de cultivares de trigo: $A-$ IRN-526-63; $B-$ Pitic-62; $C$ - IAC-8 (Ensaio Regional Paulista de Cultivares de Trigo. Cruzália, SP, 1971).

JAC-7 - Originou-se da progênie 16433, que por sua vez foi obtida por seleção, realizada na Estação Experimental de Capão Bonito, em 1956, do cruzamento entre a progênie 7124 e o cultivar Ponta Grossa I (A 7124 já foi descrita anteriormente).

LAC-8 - Originou-se da progênie 17521, resseleção do híbrido 369 , realizada na Estação Experimental de Capāo Bonito. O hibrido 369 é produto de um programa de cruzamentos múltiplos, que envolveu os cultivares Kenya 55, Heana, Gigante Inglês, Kapli e Saloio, realizado também em Campăo Bonito (figura 1). 
Os ensaios de cultivares de trigo foram plantados em condição de sequeiro. Como delineamento estatístico utilizou-se o reticulado balanceado $3 \times 3$ com quatro repetições por local.

Foram retiradas amostras compostas dos solos dos locais estudados, anteriormente ao plantio, cujos resultados analíticos $\left({ }^{4}\right)$ foram os seguintes:

\begin{tabular}{|c|c|c|c|}
\hline DETERMINAÇÕES & Tatui & Tietê & Paranapanem \\
\hline $\mathrm{pH}$ int $\ldots \ldots \ldots \ldots$ & 6,00 & 5,50 & 5,20 \\
\hline $\mathrm{C} \% \quad \ldots \ldots \ldots \ldots \ldots$ & 1,10 & 0,90 & 2,20 \\
\hline $\mathrm{K}+{ }^{(5)} \quad \ldots \ldots \ldots \ldots \ldots$ & 0,40 & 0,40 & 0,50 \\
\hline $\mathrm{Ca}^{2+}+\mathrm{Mg}^{2+}$ (s) $\ldots$ & 4,20 & 2,90 & 5,20 \\
\hline $\mathrm{PO}_{4}^{3-}$ (6) $\quad \ldots \ldots \ldots$ & 0,14 & 0,10 & 0,04 \\
\hline $\mathrm{Al}^{\mathrm{s}}+$ (5) $^{(5)} \ldots \ldots \ldots \ldots$ & - & - & 1,70 \\
\hline DETERMINAÇÕES & Capăo Bonito & Campinas & Pindorama \\
\hline $\mathrm{pH}$ int $\quad \ldots \ldots \ldots \ldots \ldots$ & 5,70 & 5,60 & 5,80 \\
\hline $\mathrm{C} \% \quad \ldots \ldots \ldots \ldots \ldots$ & 1,85 & 1,10 & 0,40 \\
\hline $\mathrm{K}^{+}{ }^{(\boldsymbol{s})} \quad \ldots \ldots \ldots \ldots \ldots$ & 0,12 & 0,18 & 0,16 \\
\hline $\mathrm{Ca}^{2+}+\mathrm{Mg}^{2+}$ (b) $\ldots$ & 3,50 & 2,50 & 1,20 \\
\hline $\mathrm{PO}_{4}{ }^{3-}{ }^{(B)} \quad \ldots \ldots \ldots \ldots$ & 0,39 & 0,09 & 0,08 \\
\hline $\mathrm{Al}^{3+}$ (s) $^{(5)} \ldots \ldots \ldots \ldots$ & - & - & - \\
\hline DETERMINAÇÕES & Monte Alegre do Sul & ltapeva & Cruzália \\
\hline $\mathrm{pH}$ int $\ldots \ldots \ldots \ldots \ldots$ & 5,10 & 5,40 & 5,60 \\
\hline$\ldots \ldots \ldots, \ldots$, & 1,15 & 1,90 & 1,10 \\
\hline $\mathrm{K}+$ (ธ) $\quad \ldots \ldots \ldots \ldots \ldots$ & 0,16 & 0,15 & 0,18 \\
\hline $\mathrm{Ca}^{2+}+\mathrm{Mg}^{2+}$ (5) $\ldots$ & 1,80 & 1,60 & 2,50 \\
\hline $\mathrm{PO}_{4^{3}}-$ (6) $^{(6)} \ldots \ldots \ldots$ & 0,05 & 0,03 & 0,09 \\
\hline $\mathrm{Al}^{\mathrm{B}+}$ (5) $^{5}$ & 0,50 & 0,80 & - \\
\hline
\end{tabular}

No ano de 1971, o Ensaio Regional Paulista de Cultivares de Trigo foi semeado no dia 16 de março, na Estação Experimental de Tatuí, em Latossolo Vermelho-Escuro orto; no dia 16 de março, na Estação Experimental de Tietê, em solo Podzólico Vermelho-Amarelo variação Laras; no dia 22 de março, na Fazenda Holambra II, situada no municipio de Paranapanema, em Latossolo Vermelho-Escuro orto; no dia 16 de março, em Campinas, na Estação Experi-

\footnotetext{
(4) Análise efetuada pela Seçáo de Fertilldade do Solo, Instituto Agronômico.

(6) e. $\mathrm{mg} / 100 \mathrm{ml}$ de solo. Teores trocáveis.

(") e. $\mathrm{mg} / 100 \mathrm{ml}$ de solo. Teor solúvel em $\mathrm{H}_{2} \mathrm{SO}, 0,05 \mathrm{~N}$.
} 
mental do Instituto Biológico do Estado de São Paulo, em solo Padzólico Vermelho-Escuro orto; no dia 23 de março, na Estação Experimental de Capão Bonito, em Latossolo Vermelho-Escuro orto; no dia 24 de março, na Estação Experimental de Pindorama, em solo Podzolizado de Lins e Marília var. Marília; no dia 6 de abril, na Estação Experimental de Monte Alegre do Sul, em solo Podzólico VermelhoEscuro orto; no dia 13 de abril, na Fazenda Primavera, localizada no município de Itapeva, em Latossolo Vermelho-Escuro orto; e no dia 26 de abril, na Fazenda São Giórgio I, municipio de Cruzália, em Latossolo Roxo.

Cada ensaio foi constituido de 36 parcelas, sendo cada parcela constituida de sete linhas de cinco metros de comprimento, espaçadas de 0,20 metro.

A semeadura foi feita na base de 40 sementes viáveis por metro de sulco, equivalendo a 1400 sementes viáveis por parcela, e por ocasiāo da colheita foran colhidas as cinco linhas centrais de cada parcela, ficando as demais como bordadura, portanto com uma área útil de $5 \mathrm{~m}^{2}$.

A adubação utilizada, aplicada totalmente no sulco de plantio, compunha-se de $30 \mathrm{~kg}$ de $\mathrm{N}, 90 \mathrm{~kg}$ de $\mathrm{P}_{2} \mathrm{O}_{5}$ e $20 \mathrm{~kg}$ de $\mathrm{K}_{2} \mathrm{O}$, por hectare, nas formas respectivamente de sulfato de amônio com 20\% de $\mathrm{N}$, superfosfato simples com $20 \%$ de $\mathrm{P}_{2} \mathrm{O}_{5}$ e cloreto de potássio com $60 \%$ de $\mathrm{K}_{2} \mathrm{O}$.

A descrição detalhada dos caracteres estudados incluindo os métodos de determinação encontram-se a seguir:

A altura das plantas de cada cultivar foi medida no campo, levando-se $\mathrm{em}$ consideração a distância do nível do solo à ponta da espiga, mantendo-se as plantas esticadas.

Foram feitas três determinações por parcela de cada um dos experimentos, considerando-se a média aritmética dos dados obtidos como a altura média do cultivar, no ano de 1971 , no Estado de São Paulo.

O periodo em dias da germinação à maturação completa de cada cultivar, foi caracterizado como ciclo. 
Para avaliação do acamamento foram computadas notas de 0 a 5 por julgamento visual no campo, onde 0 representava ótima resistência, e 5 , suscetibilidade completa, isto é, $100 \%$ das plantas acamadas.

A ocorrência de ferrugem do colmo (Puccinia graminis Pers. forma sp. tritici Eriks. et E. Henn.), ferrugem da folha (Puccinia recondita Rob. ex Desm.) e helmintosporiose (Helminthosporium sativum Pam., King. et Bakk.) foi avaliada levando-se em conta a área da planta atacada, em porcentagem, e os tipos de reação ou de infecção (6), de acordo com a descrição seguinte:

0 - imune: não há sinal de infecçăo na planta; às vezes podem ocorrer minúsculas pontuaçōes amareladas;

1 - resistente - R: uredossoro minúsculo, rodeado de área necrótica;

2 - moderadamente resistente - MR: uredossoro pequeno, geralmente dentro de uma ilha verde rodeada de bordo clorótico ou necrótico;

3 - moderadamente suscetivel - MS: uredossoro médio, coalescendo raramente, sem necrose, mas com a área clorótica geralmente presente;

4 - suscetivel - S: uredossoro grande, coalescente, sem necrose e sem clorose;

$X$ - mesotética: ocorrem diversos tipos de reação, apesar de o isolamento estar puro.

O comprimento da espiga, medido do ponto de início da ramificação até o seu ápice (5), número de espiguetas por espiga, número de grãos por espiga, número de grãos por espigueta e peso de 1000 grãos são caracteres de importância, pois acham-se relacionados com a produção dos cultivares. O número de amostras utilizadas na avaliação desses caracteres foi de 50 inflorescências para cada cultivar (5).

\section{3 - RESULTADOS E DISCUSSAO}

A germinação das sementes nas diferentes parcelas, nos nove ensaios, foi muito boa.

As produções médias por experimento e média geral, em $\mathrm{kg} / \mathrm{ha}$, acham-se no quadro 1 . 


\begin{tabular}{|c|c|c|c|c|c|c|c|c|c|c|c|c|c|}
\hline 莒 & $\sum_{\infty}^{D}$ & న్ & ళ్లి & 5 & ঙ̛. & $\begin{array}{l}\infty \\
\text { O } \\
\text { O }\end{array}$ & $\vec{\Phi}$ & \& & 怘 & $\underset{\Xi}{\Xi}$ & 1 & 1 & 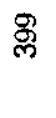 \\
\hline 兽焉 & $\stackrel{9}{8}$ & 雳 & $\frac{10}{ \pm}$ & 8 & ్ㅗㄹ & $\stackrel{8}{=}$ & 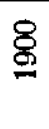 & $\underset{\Xi}{\Xi}$ & 8 & $\stackrel{N}{N}$ & $\begin{array}{l}\text { 苫 } \\
\text { i }\end{array}$ & $\frac{0}{\infty}$ & $\Re_{0}^{10}$ \\
\hline 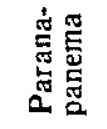 & $\stackrel{5}{50}$ & $\stackrel{\text { 怘 }}{\stackrel{\text { g }}{\leftrightarrows}}$ & $\stackrel{\text { 怘 }}{\mathrm{S}}$ & $\underset{్}{\stackrel{8}{2}}$ & $\underset{\infty}{\infty}$ & 灾 & $\stackrel{\infty}{=}$ & $\stackrel{20}{g}$ & $\stackrel{ }{5}$ & 宫 & $\stackrel{*}{\stackrel{*}{g}}$ & $\begin{array}{l}8 \\
6 \\
-1\end{array}$ & $\stackrel{9}{7}$ \\
\hline 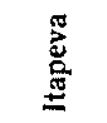 & $\frac{5}{50}$ & $\stackrel{10}{2}$ & ભু & $\stackrel{\ominus}{\cong}$ & 兽 & 号 & 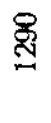 & $\underset{\infty}{\mathbb{R}}$ & 念 & 迠 & 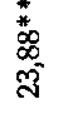 & $\begin{array}{l}\text { 웅 } \\
\text { क }\end{array}$ & $\stackrel{\mathscr{\aleph}}{\underset{\sim}{*}}$ \\
\hline 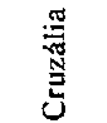 & 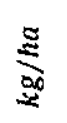 & $\stackrel{?}{2}$ & 号 & 옹 & 尽 & $\stackrel{n}{\sigma}$ & $\frac{⿱ 乛 ⿻}{7}$ & ళ్ర & 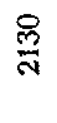 & $\stackrel{10}{\mathscr{\infty}}$ & 5 & 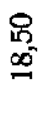 & $\stackrel{\substack{\infty \\
\infty}}{\infty}$ \\
\hline 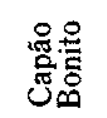 & 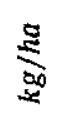 & $\underset{⿱}{\infty}$ & $\stackrel{\mathscr{D}}{=}$ & ஜ్ & $\frac{5}{\sigma}$ & $\stackrel{\text { N }}{\Xi}$ & 苞 & 5 & 约 & $\bar{\sigma}$ & m & 온 & $\underset{R}{\mathcal{O}}$ \\
\hline 宫惫 & 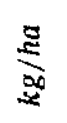 & $\underset{g}{\stackrel{g}{\Xi}}$ & త్ & 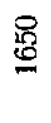 & $\stackrel{6}{0}$ & ํㅗำ & $\begin{array}{l}\text { ळ } \\
\text { ల్ }\end{array}$ & $\underset{\mathbb{్}}{\mathbb{\Xi}}$ & $\begin{array}{l}10 \\
08 \\
0\end{array}$ & $\stackrel{\mathscr{\infty}}{\stackrel{\infty}{ \pm}}$ & $\stackrel{9}{9}$ & $\frac{q}{5}$ & \pm \\
\hline 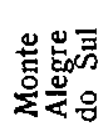 & 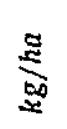 & $\stackrel{17}{\stackrel{్}{J}}$ & $\stackrel{R}{E}$ & 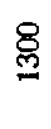 & $\stackrel{8}{2}$ & 总 & $\stackrel{\varrho}{\Xi}$ & $\stackrel{\mathscr{m}}{\dddot{m}}$ & 员 & 옹 & $\frac{*}{n}$ & $\frac{8}{i}$ & 8 \\
\hline 声 & $\underbrace{5}_{0}$ & ్ㅐㅇ & $\stackrel{12}{\Xi}$ & $\underline{8}$ & ళ్రి & 8 & $\stackrel{8}{8}$ & జ్ & 尚 & ஜ্চ & 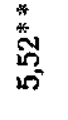 & $\frac{8}{i}$ & $\frac{n}{n}$ \\
\hline 总 & $\sum_{0}^{0}$ & 올 & $\frac{10}{6}$ & ঙ্ল్ & 蛋 & $\frac{10}{6}$ & 8 & $\stackrel{\mathscr{O}}{\stackrel{\Theta}{=}}$ & $\stackrel{n}{m}$ & $\stackrel{\infty 2}{=}$ & $\begin{array}{l}* \\
\stackrel{*}{*} \\
\infty\end{array}$ & $\begin{array}{l}\text { 员 } \\
\text { ๙ิ }\end{array}$ & 苛 \\
\hline$\frac{\stackrel{5}{7}}{3}$ & & $\underset{⿱ 亠 䒑}{\stackrel{I}{I}}$ & $\begin{array}{l}0 \\
0 \\
J\end{array}$ & $\frac{N}{1}$ & $\ddot{m}$ & 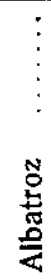 & 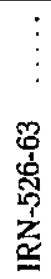 & 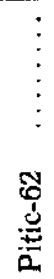 & $\begin{array}{c}\vdots \\
\vdots \\
\text { J }\end{array}$ & 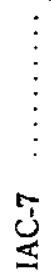 & $\begin{array}{c}\vdots \\
\vdots \\
\vdots \\
\vdots\end{array}$ & $\begin{array}{c}\vdots \\
\vdots \\
0 \\
0\end{array}$ & 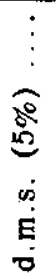 \\
\hline
\end{tabular}


Os resultados da análise estatística conjunta dos nove experimentos (quadro 2) mostram que houve diferença altamente significativa entre os cultivares e entre os locais estudados. A interação cultivar x local também foi altamente significativa, mostrando que os cultivares apresentam diferente comportamento em função dos locais.

QUADRo 2. - Análise da variância conjunta dos dados de produçāo de trigo em grãos $(\mathrm{kg} / \mathrm{ha})$ obtidos em nove ensaios de cultivares, instalados em 1971 e resumidos no quadro 1

\begin{tabular}{c|r|r|r|r}
\hline Fonte de variaçăo & \multicolumn{1}{|c|}{ S.Q. } & G.L. & Q.M. & \multicolumn{1}{c}{ F } \\
\hline Cultivar $\ldots \ldots \ldots \ldots \ldots$ & 27610800 & 8 & 3451350 & $14,45^{* *}$ \\
Local $\ldots \ldots \ldots \ldots \ldots$ & 7915100 & 8 & 989388 & $3,57^{* *}$ \\
Cultivar x local $\ldots \ldots \ldots$ & 17736200 & 64 & 277128 & $3,65^{* *}$ \\
Resíduo médio $\ldots \ldots \ldots$ & & 216 & 76008 & \\
\hline
\end{tabular}

Aplicando-se o teste de Tukey na comparação das médias gerais dos cultivares, ao nível de 5\%, verificou-se que: S-12 e IRN-526-63 foram superiores em produção ao S-33. Os cultivares cujas produções médias atingiram níveis melhores foram S-12, IRN-526-63, Pitic-62, BH-1146 e IAC-7.

Considerando os ensaios instalados em Latossolo Vermelho-Escuro orto (Tatuí, Capão Bonito, Parapanema e Itapeva), verificou-se que os cultivares BH-1146, Pitic-62, IRN-526-63 e S-12 foram os que apresentaram melhores produções; os cultivares IAC-5, IAC-7 e IAC-8, com produções intermediárias; e os cultivares Albatroz e S-33, com produções inferiores.

Os cultivares Pitic-62, IRN-526-63 e IAC-7 apresentaram produçōes superiores a $2000 \mathrm{~kg} / \mathrm{ha}$, no ensaio localizado na Fazenda São Giórgio I, município de Cruzália, em Latossolo Roxo, ao passo que o cultivar BH-1146, que era o mais cultivado na região, produziu $1770 \mathrm{~kg} / \mathrm{ha}$.

Em condição de baixada tem-se o ensaio localizado na Estação Experimental de Monte Alegre do Sul, onde o cultivar IAC-5 apresentou boa adaptação, produzindo $1770 \mathrm{~kg} / \mathrm{ha}$, seguido dos cultivares IRN-526-63 e Pitic-62, com 1410 e $1335 \mathrm{~kg} / \mathrm{ha}$, respectivamente. 
Observando o quadro 3 , verifica-se que os cultivares $\mathrm{S}-12$ e IRN-526-63 apresentaram resistência à ferrugem do colmo; S-33, IAC-8, IAC-5, Albatroz, Pitic-62 e IAC-7, média resistência; e o cultivar $\mathrm{BH} 1146$, alta suscetibilidade à ferrugem do colmo.

Entre os cultivares estudados, IAC-8 foi aquele que apresentou maior resistência à ferrugem da folha, ao passo que os demais apresentaram-se com média suscetibilidade.

Considerando as observações de acamamento, verifica-se que os cultivares IRN-526-63, Pitic-62 e BH-1146 apresentaram resistência; IAC-5, S-12, S-33, IAC-7 e IAC-8, média suscetibilidade; e o cultivar Albatroz, alta suscetibilidade.

\section{4 - CONCLUSŐES}

a) Os cultivares S-12, IRN-526-63, Pitic-62, BH-1146, IAC-5 e IAC-7 apresentaram boa adaptação e produção nas diferentes regiões do Estado de Săo Paulo. O cultivar S-12 somente diferiu estatisticamente quanto à produção, pelo teste de Tukey a $5 \%$, na média dos nove experimentos, dos cultivares Albatroz e S-33. O cultivar IRN526-63 somente diferiu estatisticamente do cultivar S-33.

b) Os cultivares S-12 e IRN-526-63 mostraram-se resistentes à ferrugem do colmo.

c) Os cultivares S-33, IAC-5, Albatroz, Pitic-62, IAC-7 e IAC-8 apresentaram boa resistência de campo à ferrugem do colmo.

d) O cultivar BH-1146 apresentou-se altamente suscetivel à ferrugem do colmo.

e) Entre os cultivares estudados, IAC-8 foi o que apresentou menor suscetibilidade a ferrugem da folha, ao passo que os demais apresentaram média suscetibilidade.

f) Os cultivares IRN-526-63, Pitic-62 e BH-1146 apresentaramse como resistentes ao acamamento. 


\begin{tabular}{|c|c|c|}
\hline & 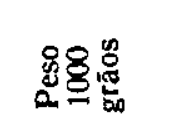 & 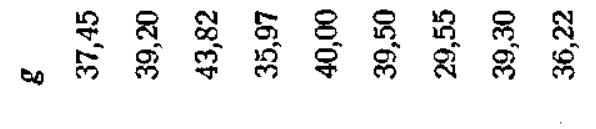 \\
\hline & 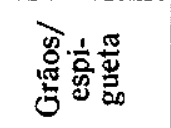 & 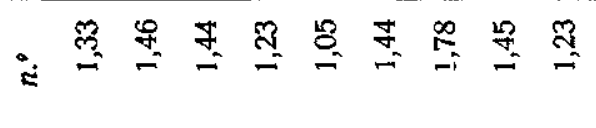 \\
\hline \multirow{9}{*}{ 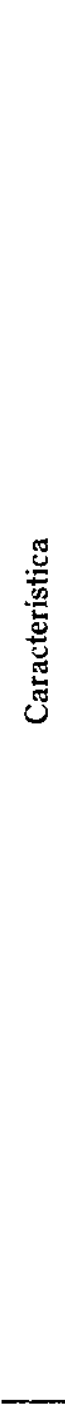 } & 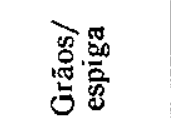 & $\therefore$ \\
\hline & 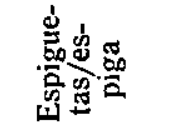 & 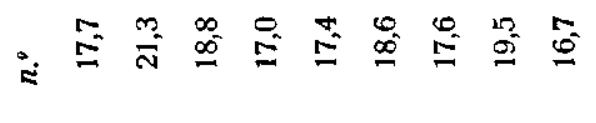 \\
\hline & 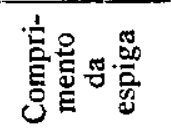 & 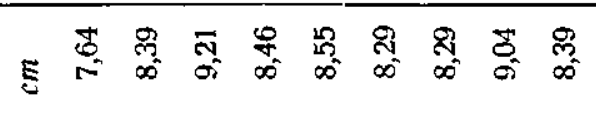 \\
\hline & 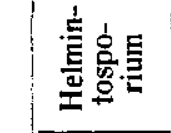 & 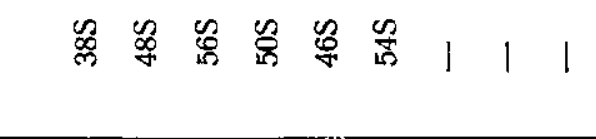 \\
\hline & 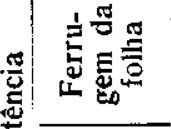 & 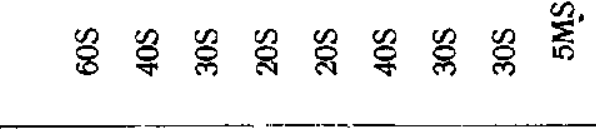 \\
\hline & : & 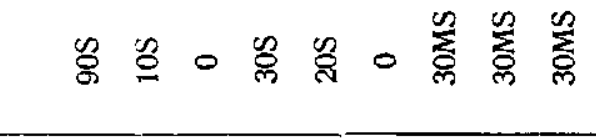 \\
\hline & 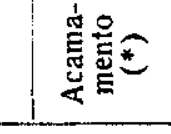 & 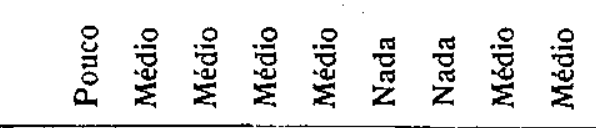 \\
\hline & $\stackrel{\circ}{0}$ & 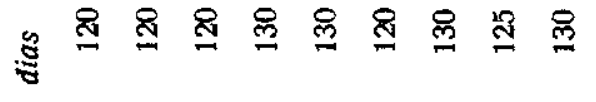 \\
\hline & 竞 & E \\
\hline & 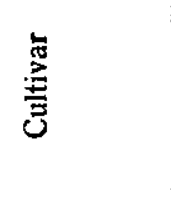 & 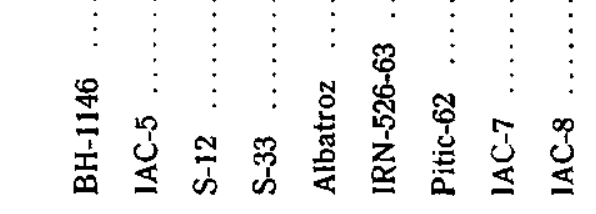 \\
\hline
\end{tabular}




\section{BEHAVIOUR OF WHEAT CULTIVARS IN DIFFERENT REGIONS OF THE STATE OF SAO PAULO WITHOUT IRRIGATION}

\section{S U M M A R Y}

Competition trials of nine cultivars of wheat (Triticum aestivum L.) were carried out without irrigation in nine different areas of the State of Săo Paulo, in 1971. The new CIMMYT cultivar IRN-526-63, created in Mexico, proved to be the best one.

This cultivar presented high yield, straight and low type of plants, early maturation, resistance to lay, to stem rust caused by Puccinia graminis tritici, and a wide adaptation range in the State.

\section{LITERATURA CITADA}

1. CAMARGO, C. E. O. Ensaios Regionais. In: Encontro sobre Triticultura. Campinas, CATI, 1971. 28p.

2. Relatório dos trabalhos desenvolvidos com trigo pela Seçăo de Arroz e Cereais de Inverno, Campinas, Instituto Agronómico, 1969, 1970 e 1971. (Não publicado)

3. ISSA, E. Trabalhos do Instituto Biológico de São Paulo. Reunião da Sub Comissão Norte da Comissăo Brasileira de Trigo. Londrina, 1971. 9 fls.

4. - Doenças do trigo, In: Encontro sobre Triticultura. Campinas, CATI, 1971. 20p.

5. LEITAO FILHO, H. F. Botânica do Trigo. In: Encontro sobre Triticultura. Campinas, CATI, 1971. 24p.

6. STAKMAN, E. C.; LEVINE, M. N. \& LOEGERING, W. Q. Identification of physiologic races of Puccinia graminis tritici. Agricultural research administration - United States Department of Agriculture. U.S.A., 1944. 27p. 